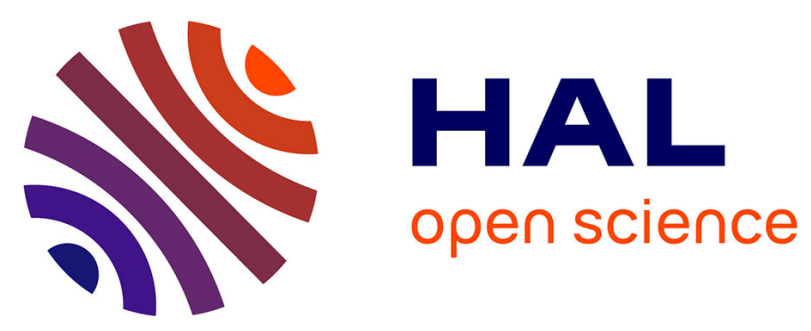

\title{
Les revues en sciences humaines et sociales à l'heure des communs
}

\author{
Odile Contat, Didier Torny
}

\section{To cite this version:}

Odile Contat, Didier Torny. Les revues en sciences humaines et sociales à l'heure des communs . Revue d'Histoire Moderne et Contemporaine, 2015, Économie et politique de l'" accès ouvert ": les revues à l'âge numérique, 62-4 bis, pp.62-70. 10.3917/rhmc.625.0062 . halshs-01278853v2

\section{HAL Id: halshs-01278853 \\ https://shs.hal.science/halshs-01278853v2}

Submitted on 10 Apr 2016

HAL is a multi-disciplinary open access archive for the deposit and dissemination of scientific research documents, whether they are published or not. The documents may come from teaching and research institutions in France or abroad, or from public or private research centers.
L'archive ouverte pluridisciplinaire HAL, est destinée au dépôt et à la diffusion de documents scientifiques de niveau recherche, publiés ou non, émanant des établissements d'enseignement et de recherche français ou étrangers, des laboratoires publics ou privés. 


\title{
Les revues en sciences humaines et sociales à l'heure des communs
}

\section{Odile Contat \& Didier Torny (InSHS, CNRS)}

\author{
Manuscrit pour la RHMC, remis le 4 novembre 2015, corrigé le 14 janvier 2016 \\ Forme publiée : http://www.cairn.info/resume.php?ID_ARTICLE=RHMC_625_0062
}

Les échanges récents autour de la loi numérique, comme les discussions ayant fait suite à la mise en place de politiques d'open access par le Conseil Européen de la Recherche ont largement contribué à publiciser, en France, des débats se déroulant à l'échelle internationale sur les transformations de l'économie politique des publications scientifiques.

Sans discuter ici la vaste littérature produite par des chercheurs en sciences documentaires, mais aussi par différentes parties prenantes (militants de l'accès ouvert, éditeurs, sociétés savantes, financeurs, instances politiques), nous rappellerons les caractéristiques de l'édition scientifique en SHS en France, puis nous décrirons les formes contemporaines d'appropriation des publications scientifiques par les éditeurs et diffuseurs. Enfin, nous analyserons différentes politiques publiques nationales et exposerons les fondements de la politique du CNRS, et plus particulièrement de l’InSHS, en matière de soutien à l'édition scientifique.

\section{La publication scientifique comme produit d'une économie publique}

Les publications scientifiques, notamment sous la forme de revues, se sont développées de manière exponentielle depuis le XVII ${ }^{\text {ème }}$ siècle : le nombre de titres, d'articles et de pages publiées n'a cessé d'augmenter (Kronick 1976). Cette progression résulte d'une diversification des communautés scientifiques et d'une extension de chacune d'entre elles, découlant de l'investissement public des Etats-Nations, d'abord en Europe Occidentale et aux États-Unis, puis à l'échelle mondiale. En effet, l'économie des revues scientifiques relève presque exclusivement de la dépense publique, à partir des différents postes que nous rappelons ci-dessous.

Premièrement, les auteurs des recherches sont très majoritairement des chercheurs payés sur fonds publics, au moins dans l'espace européen. Deuxièmement, lorsque les recherches donnent lieu à un financement, celui-ci est très majoritairement public: agences nationales, fonds européens, collectivités territoriales, opérateurs publics divers apportent leur contribution. A contrario, les financements privés ne conduisent pas nécessairement à des publications, notamment pour permettre l'usage des dispositifs de propriété intellectuelle. Troisièmement, le travail d'édition est encore une fois largement public : les membres des comités de rédaction, les reviewers, mais aussi parfois les personnels en charge de la production de la revue, sont des agents publics. Enfin, quatrièmement, les acquéreurs des publications sont publics: bibliothèques universitaires et organismes de recherche sont les clients payants quasiment exclusifs des éditeurs et diffuseurs de science. Ces constats maintes fois répétés à l'échelle internationale ont été confirmés pour la situation française en SHS par une récente étude (Contat \& Gremillet 2015), dont nous exposons cidessous les principaux résultats. 
Elle montre que l'ensemble des contenus scientifiques des articles de revues sont produits et expertisés par des chercheurs payés, dans leur écrasante majorité, par la puissance publique. Dans neuf cas sur dix, l'ensemble du travail éditorial de production d'une revue en SHS (travail sur le texte, depuis l'appel à contribution jusqu'au bon à composer, et dans près de six cas sur dix jusqu'au bon à tirer) est assuré par la puissance publique au sein des institutions et des unités de recherche producteurs de revues. L'éditeur privé, lorsqu'il intervient, prend en charge, le plus souvent, l'impression, la diffusion et la distribution et en tire un revenu commercial loin d'être toujours partagé avec l'institution publique. La partie la plus importante du coût éditorial d'un article publié est bien celle du salaire de secrétariat de rédaction : le temps moyen nécessaire pour les tâches de secrétariat de rédaction est de 10,5 mois par revue et par an, le coût salarial moyen par revue est de $42000 €$. La part des coûts nécessaires à l'impression, la diffusion et la distribution n'est donc pas prédominante par rapport aux coûts éditoriaux du salaire du secrétariat de rédaction dans le cas des revues SHS.

Même si cette étude n’inclut pas les coûts indirects du travail scientifique - écriture des articles, lecture et évaluation, elle souligne bien, d'une part, le coût élevé des publications scientifiques, d'autre part, leur prise en charge quasi-exclusive par des opérateurs publics ou parapublics dans ces disciplines. Ce double constat légitime l'intervention des opérateurs publics dans l'organisation de l'édition scientifique. Mais quelles options s'offrent à eux, hors de la mise à disposition attestée de ressources et de personnes? Pour les comprendre, il faut d'abord considérer la situation d'appropriation de ces publications et les réponses déjà apportées par différentes communautés scientifiques.

\section{L’appropriation des communs par les éditeurs et diffuseurs}

Les arguments déployés en faveur de l'accès ouvert aux publications scientifiques sont nombreux : favoriser la discussion scientifique, accélérer l'innovation, alimenter des débats publics avec le véritable état des connaissances, rendre visibles des recherches demeurées obscures, etc. Mais la conception politique sous-jacente est celle d'une désignation de ces publications comme biens communs (Suber, 2007), ne devant pas faire l'objet d'appropriations exclusives. L'électronisation des publications, réellement mise en œuvre à partir de 1995, a rendu visible la privatisation de ces communs, notamment par les éditeurs scientifiques dominants, qui distillent l'accès à ces ressources contre des sommes toujours plus importantes. Cela entraîna paradoxalement la restriction de leur diffusion, certaines institutions ne pouvant plus ou ne souhaitant plus financer de tels accès, « crise des périodiques » sur laquelle les sciences documentaires états-uniennes ont commencé à alerter il y a déjà vingt ans ${ }^{1}$.

Mais observer la limitation de la diffusion par une barrière économique ne permet pas de saisir l'ensemble des dispositifs ayant conduit à une appropriation progressive des publications scientifiques. Celle-ci commençait dès la signature de contrats d'édition qui ont des caractéristiques très particulières : en effet, les auteurs cèdent généralement l'exclusivité de leur travail à des éditeurs/diffuseurs sans qu'eux-mêmes ou leurs institutions ne soient rétribués ${ }^{2}$. De plus, cette cession limite le plus souvent, voire interdit, toute autre forme de réutilisation des articles. Elle se poursuivait par la généralisation d'un service d'accès toujours temporaire plutôt que par la possession définitive des articles par les institutions acheteuses. Enfin, l'existence d'un " pouvoir de marché » exercé par les plus gros éditeurs conduisait à des achats groupés, sous la forme de

\footnotetext{
${ }^{1}$ Jean-Claude Guédon a joué un rôle important dans la diffusion en français de ces débats, voir (Guédon 2001)

${ }^{2}$ À l'exception sans doute de certaines revues juridiques. La situation est relativement différente pour les ouvrages.
} 
bouquets de revues, plutôt qu'à un choix d'abonnements fondé sur de strictes raisons de politique scientifique et documentaire ${ }^{3}$.

Alors que ces formes d'appropriation faisaient l'objet d'une dénonciation croissante, différents modes d'action ont été proposés et mis en œuvre par des communautés scientifiques. On peut notamment citer le mouvement de «libération » des revues, consistant à rompre les liens avec certains éditeurs, à recréer des revues, ainsi que différents boycotts d'éditeurs, en tant qu'auteur, membre de comité de rédaction ou reviewer, le plus célèbre d'entre eux étant celui visant Elsevier dans le cadre de la pétition « The cost of knowledge » en $2012^{4}$. C'est encore la création de formats inédits, purement électroniques, de nouvelles formes d'évaluation par les pairs, celle-ci se déroulant non plus avant, mais après la diffusion ${ }^{5}$, comme dans le cas du portail Episciences ${ }^{6}$. C'est enfin la promotion de nouvelles formes de propriété intellectuelle, que ce soit par la diffusion de contratstypes plus favorables aux auteurs ou par de réelles inventions, au premier rang desquelles les licences Creative Commons ${ }^{7}$.

Parmi toutes ces initiatives, deux dispositifs d'accès ouvert aux publications ont connu un grand succès : la généralisation des archives ouvertes ${ }^{8}$ d'une part, l'invention de nouveaux modèles économiques ne reposant pas sur l'abonnement d'autre part. Si le mouvement pour les archives ouvertes a d'abord touché la physique, il a connu des développements importants en SHS, notamment autour de RePEC (Research Papers in Economics), archive décentralisée inventée par des économistes au milieu des années 1990 et comptant en 2015 près de 700000 manuscrits de travail et plus d'un million d'articles publiés. Elles ont largement permis la circulation des manuscrits et leur discussion scientifique avant, pendant et après le processus de publication certifié, indépendamment de l'existence d'accès payants aux articles. Concernant les nouveaux modèles économiques, si les sciences de la vie ont été les moteurs des dispositifs d'auteur-payeur à travers les éditeurs BMC et PLOS, de nombreuses initiatives ont existé en SHS. Ainsi, tout récemment, un groupe de linguistes a lancé LingOA, revue soutenue financièrement par six universités néerlandaises et l'agence de financement NWO (Nederlandse Organisatie voor Wetenschappelijk Onderzoek). Au service de ce projet, les membres des comités de rédaction de cinq revues scientifiques majeures en linguistique se proposent soit de quitter leur éditeur, soit de renégocier leur collaboration.

Le but de leur démarche est d'améliorer l'accessibilité des résultats de la recherche tout en étant moins dépendant des éditeurs commerciaux. Dans LingOA, les chercheurs conserveront leur droit d'auteur et les frais qui leur seront demandés seront inférieurs à ceux des éditeurs commerciaux. La plateforme Open Library of Humanities ${ }^{9}$ sert de support à ce programme.

Si ces initiatives ont redéfini les formes de diffusion scientifique et contribué à modifier le paysage de l'édition, elles n’ont pas entièrement résolu la question de l'appropriation des communs. En effet, les éditeurs commerciaux se sont adaptés et ont notamment développé le modèle de la revue hybride, proposant aux auteurs de rendre leur texte accessible librement contre une somme de plusieurs milliers d'euros tout en conservant la nécessité de l'abonnement pour accéder aux articles non «financés» par cette voie. Tout cela a conduit de nombreux pays à définir des politiques

\footnotetext{
${ }^{3}$ Voir le rapport commandé par la Commission Européenne (Dewatripont \& al. 2006).

${ }^{4}$ http://thecostofknowledge.com/

5 Sur les transformations contemporaines de l'évaluation par les pairs pour les revues, et l'introduction des lecteurs comme évaluateurs voir (Pontille \& Torny 2015).

${ }^{6}$ http://www.episciences.org

7 Sur les modalités de création et les objectifs de ces licences, voir (Bourcier \& Dulong de Rosnay 2004)

${ }^{8}$ Une archive ouverte est un réservoir où sont déposés les travaux issus de la recherche scientifique et de l'enseignement pour un accès libre et immédiat (voie verte/green du libre accès - la voie dorée/gold désignant les revues en accès libre)

${ }^{9}$ https://www.openlibhums.org/
} 
publiques en matière d'accès aux publications scientifiques, souvent fondés sur des dispositifs inventés par des communautés scientifiques.

\section{Des politiques publiques pour la diffusion scientifique}

Nous ne pouvons dans ce court texte décrire l'ensemble de ces politiques publiques, qui différent sensiblement d'un pays à l'autre ${ }^{10}$. Nous avons choisi de distinguer quatre principaux types de dispositifs d'action publique avec leurs objectifs propres.

Premièrement, on observe la construction d'archives ouvertes centralisées, destinées à recueillir l'ensemble des productions scientifiques consécutives à un financement public. C'est notamment le cas de l'archive états-unienne PubMed Central, de sa déclinaison européenne Europe PMC, dans le domaine des sciences de la vie, modèle aujourd'hui repris par exemple par l'Espagne ou l'Argentine. Les publications y sont déposées après une période dite d'embargo ${ }^{11}$, typiquement six ou douze mois, après laquelle les éditeurs perdent de fait l'exclusivité de la diffusion. Il faut souligner que ces dépôts ne changent en rien la question du coût des abonnements, les éditeurs en sciences de la vie, et au premier rang Elsevier, n'ayant pas du tout souffert du succès de PubMed Central. A ces dépôts de financeurs de projets, s'ajoutent des archives ouvertes d'employeurs : de nombreuses universités à l'étranger obligent au dépôt dans leurs archives ouvertes, des publications de leur personnel. C'est également le cas de l'Inria et de l'Ifremer en France.

Deuxièmement, on constate le développement de politiques de soutien à la publication en libre accès, notamment par la prise en charge des frais de type auteur-payeur par le financeur de la recherche en Grande-Bretagne, aux Pays-Bas ou sur certains programmes européens. Troisièmement, on voit apparaître un droit légal d'utilisation secondaire pour les auteurs des articles, notamment en Allemagne, sur la base du droit d'auteur, lui aussi assorti d'une période d'embargo. Quatrièmement, le Brésil avec SciELO et de nombreux pays hispanophones avec Redalyc ont littéralement construit des infrastructures publiques d'édition scientifique électronique, permettant d'accueillir et de diffuser des milliers de revues en portugais et en espagnol, notamment dans les disciplines de SHS.

Ce foisonnement d'initiatives provenant d'établissements publics ou des pouvoirs politiques a pour objectif l'accès et la conservation des informations scientifiques issues de la recherche financée par des fonds publics. C'est la raison pour laquelle la Commission Européenne a recommandé en 2013 aux États-Membres de définir leurs propres politiques afin de veiller à ce que «les publications soient librement accessibles dans les meilleurs délais, de préférence immédiatement et, dans tous les cas, au plus tard six mois après leur date de publication, et au plus tard douze mois pour les publications dans les domaines des sciences sociales et humaines ${ }^{12}$.

C'est cette recommandation qui est aujourd'hui en discussion en France dans le cadre de la loi numérique, et notamment son article concernant le libre accès aux publications scientifiques de la recherche publique. L'organisation inédite d'une consultation en ligne sur ce texte avant sa discussion au Parlement a particulièrement mobilisé la communauté scientifique (1840 votes) et proposé des modifications au texte initial de cet article qui prévoyait un délai d'embargo de douze mois hors SHS et de vingt-quatre mois pour les publications en SHS.

10 On trouvera un panorama à jour sur le site Open access de l'INIST http://openaccess.inist.fr/?Point-sur-les-politiques-en-faveur

${ }^{11}$ Embargo : durée contractuelle pendant laquelle l'auteur ne peut pas déposer un article dans une archive ouverte.

${ }^{12}$ Recommandation de la Commission sur l'accès et la conservation des informations scientifiques issues de la recherche financée par des fonds publics, 17 juillet 2012. http://ec.europa.eu/research/science-society/document_library/pdf_06/recommendation-access-andpreservation-scientific-information_fr.pdf 


\section{La politique du CNRS et la position de l'InSHS}

Ces débats permettent d'exposer la position de l'InSHS, inscrite dans la politique du CNRS, mais également coordonnée avec de nombreux acteurs de l'enseignement supérieur et de la recherche. Ainsi, la Conférence des Présidents d’Universités (CPU), l'Association des directeurs et des personnels de direction des bibliothèques universitaires (ADBU), la Direction de l'information scientifique et technique du CNRS, le Conseil national du numérique ont tous pris position en faveur de la diffusion en accès ouvert des résultats de la recherche scientifique. Le Conseil scientifique du CNRS a récemment affirmé que «1) la science est un bien commun de l'humanité [...], 2) le libre accès aux résultats de l'activité scientifique (publications, données de la recherche, métadonnées, services à valeur ajoutée) ne saurait être entravé sans remettre en cause le développement même de la science $"{ }^{13}$. Au sein du CNRS, l’InSHS est particulièrement attaché à la mise en place d'un large libre accès aux travaux scientifiques financés sur fonds publics. L'Institut soutient notamment depuis très longtemps, le dépôt en archives ouvertes des travaux des chercheurs financés sur fonds publics, et incite ses chercheurs à déposer dans HAL-SHS, qui vient de fêter ses dix ans.

L'importance financière du soutien de l'Institut aux revues, avec un budget de 140000 euros annuels, en fait un instrument de pilotage par rapport à cet objectif de diffusion. Ainsi, les revues souhaitant être aidées ont progressivement diminué leur barrière mobile ${ }^{14}$ sur les portails de diffusion, en passant de quatre à trois ans, ou de trois à deux ans. On sait depuis longtemps que l'accès libre permet d'augmenter la lecture de ces textes et leur citation, et une étude récente de l'Institut des politiques publiques, commandée par le ministère de la Recherche, montre de plus qu'un article seulement diffusé par voie d'abonnement subit un préjudice de visibilité qui est impossible à rattraper à partir de 12 mois de barrière mobile. Un article soumis à paiement ne parvient pas à retrouver le lectorat perdu au moment où il passe enfin en accès ouvert, et ce handicap est d'autant plus lourd que la durée de la barrière mobile est longue (Bacache-Beauvallet \& al., 2015).

En conséquence, les revues n’ayant pas de diffusion numérique pour les années les plus récentes et les revues ayant une diffusion numérique entièrement payante ne peuvent être éligibles au soutien de l'InSHS. La campagne d'aide financière aux revues est aussi un levier important de diffusion des bonnes pratiques dans leur production éditoriale et leur diffusion. Outre les critères de transparence et d'ouverture dans l'organisation des instances des revues (une durée de mandat prévue pour le rédacteur ou la rédactrice en chef) et l'évaluation des articles (un expert extérieur au moins), il s’agit pour l'Institut de promouvoir des modes de production électroniques professionnels (rapidité de la production, utilisation de la chaîne XML TEI) et d'inciter les revues à une (re)prise en main de leur diffusion (référencement, diffusion multi-portail). Dans cette perspective d'amélioration des pratiques éditoriales des revues françaises en SHS, la présence d’un personnel ingénieur, secrétaire de rédaction ou d'édition, est de loin la meilleure ressource car il permet une véritable professionnalisation des équipes éditoriales. L'investissement de l'InSHS est en effet d'importance unique en la matière puisque 89 revues françaises en SHS bénéficient de l'équivalent temps plein de 54 agents publics ${ }^{15}$, soit un apport annuel supérieur à trois millions d'euros.

Enfin, comme dans d'autres pays, il faut mentionner que l'Institut a largement contribué à la construction de plateformes dédiées aux revues en SHS dans le cadre du Centre pour l'édition électronique ouverte, en partenariat avec Aix-Marseille Université, l'EHESS et l'Université

\footnotetext{
13 Motion du Conseil Scientifique du CNRS, 25 septembre 2015 http://www.cnrs.fr/dist/zoutils/documents/Motion\%20Conseil\%20Scientifique\%20CNRS\%2025092015.pdf

${ }^{14}$ Barrière mobile : durée pendant laquelle les articles de la revue ne sont pas en accès ouvert sur le site du diffuseur (accès sous abonnement)

${ }^{15}$ D'autres institutions de l'ESR, universités et grands établissements contribuent également, à leur échelle, à soutenir les revues en SHS par la mise à disposition de personnel IST.
} 
d’Avignon. La plateforme Revues.org héberge, fin 2015, 411 revues en SHS, assurant leur très large diffusion, 70\% d'entre elles étant aujourd'hui en accès totalement ouvert, sans aucune barrière mobile.

\section{Quel modèle économique pour les revues en SHS ?}

Nous avons montré dans ce court article que les revues en SHS, et notamment celles éditées en France, sont le produit d'une économie quasiment exclusivement publique. Il est donc légitime que les opérateurs publics définissent des politiques, en partenariat avec les revues, quant aux usages de cet argent public, et s'opposent à une appropriation indue des contenus scientifiques. Néanmoins, certains critiques s'inquiètent de ces interventions publiques et craignent même qu'elles ne mettent en péril les revues.

Nous avons détaillé l'importance des investissements au sein de l'InSHS, mais il faut aussi souligner le rôle qu'a joué la plateforme Persée dans la mise à disposition des fonds patrimoniaux de plus de 200 revues, qui sont en conséquence bien plus consultés aujourd'hui qu'ils ne l'ont jamais été. Dans chaque cas, il s'est agi de favoriser la diffusion, la lecture, le débat scientifique, au profit des revues et des communautés scientifiques. De façon similaire aux expériences internationales antérieures ${ }^{16}$, la politique de diminution des barrières mobiles n'a absolument pas entamé les revenus des revues puisqu'elle n’a pas entraîné de diminution des abonnements. Aujourd'hui, la mise en œuvre des recommandations européennes ne les menace pas davantage (Lemercier \& Dacos, 2015).

En revanche, le modèle économique fondé sur des abonnements papier se meurt : toutes les revues, et surtout les plus diffusés via ce support, constatent une érosion de leur lectorat et de leur revenu par ce support. Les données actuelles dont nous disposons montrent que de nombreuses revues publient à perte leur édition papier, le financement provenant en fait de l'édition électronique. Comme dans d'autres disciplines, il s'agit aujourd'hui d'adopter des modèles économiques adaptés à cette forme de production: l'InSHS soutient bien évidemment le Centre pour l'édition électronique ouverte (CLEO) et son modèle freemium permettant d'assurer un revenu aux revues tout en maximalisant leur référencement et leur diffusion, mais d'autres sont sans doute à inventer. Aussi, dans cette période de transition, l'InSHS continuera à accompagner les revues vers cette diffusion en libre accès. Si les connaissances et les revues que nous produisons collectivement ont un coût, leur valeur réside dans le fait de les partager, et l'accès libre ne fait qu'y contribuer.

\footnotetext{
${ }^{16}$ Une des conclusions du projet PEER Executive Partners Achievements \& Reflections est que « rien ne prouve que l'auto-archivage ait des effets nuisibles sur la viabilité des revues » http://www.peerproject.eu/fileadmin/media/presentations/PEER-Executive_Partners_Statements_29_May_2012.pdf
} 


\section{Bibliographie}

Maya Bacache-Beauvallet, Françoise Benhamou, Marc Bourreau, Les revues de sciences humaines et sociales en France: libre accès et audience, Rapport IPP nº11 - Juillet 2015, http://www.ipp.eu/publication/juillet-2015-revues-sciences-humaines-et-sociales-shs-en-francelibre-acces-et-audience/

Danièle Bourcier et Mélanie Dulong De Rosnay, International commons at the digital age. La création en partage, Paris, Romillat, 2004. https://hal.archives-ouvertes.fr/hal-00666304/document

Odile Contat et Anne-Solweig GremiLlet, « Publier : à quel prix ? Étude sur la structuration des coûts de publication pour les revues françaises en SHS ", Revue française des sciences de l'information et de la communication [En ligne], 7 | 2015, mis en ligne le 13 octobre 2015. http://rfsic.revues.org/1716

Mathias DEWATRIPONT et al., Study on the economic and technical evolution of the scientific publication markets in Europe, 2006 (https://dipot.ulb.ac.be/dspace/bitstream/2013/9545/1/md0005.pdf).

Jean-Claude GuEDON, A l'ombre d'Oldenburg : bibliothécaires, chercheurs scientifiques, maisons d'édition et le contrôle des publications scientifiques communication au congrès de l'ARL, Toronto, Mai 2001, https://halshs.archives-ouvertes.fr/halshs-00395366/document.

David A KronicK, A History of Scientific and Technical Periodicals: The Origin and Development of the Scientific and Technical Press, 1665-1790, 2nd ed. Metuthen, N.J. Scarecrow Press, 1976

Claire LEMERCIER et Marin DACOS ; Pourquoi il faut distinguer clairement les archives ouvertes et l'édition électronique ouverte, billet du blog L'édition électronique ouverte, publié le 13 octobre 2015 http://leo.hypotheses.org/12523

David PonTILle et Didier ToRnY, " From Manuscript Evaluation to Article Valuation: The Changing Technologies of Journal Peer Review ", Human Studies, 38-1, 2015, p. 57-79. https://tel.archives-ouvertes.fr/hal-01143310/document

Peter Suber, Creating an Intellectual Commons through Open Access, in Charlotte HEss et Elinor Ostrom (eds.), Understanding Knowledge as a Commons: from Theory to Practice, MIT Press, 2007, p. 171-208 http://www.wtf.tw/ref/hess_ostrom_2007.pdf\#page=186. 